\title{
ON KNOTS WITH NONTRIVIAL INTERPOLATING MANIFOLDS
}

\author{
BY \\ JONATHAN SIMON $\left({ }^{1}\right)$
}

\begin{abstract}
If a polygonal knot $K$ in the 3-sphere $S^{3}$ does not separate an interpolating manifold $S$ for $K$, then $S-K$ does not carry the first homology of either closed component of $S^{3}-S$. It follows that most knots $K$ with nontrivial interpolating manifolds have the property that a simply connected manifold cannot be obtained by removing a regular neighborhood of $K$ from $S^{3}$ and sewing it back differently.
\end{abstract}

0. Introduction. A polygonal knot $K$ in the 3-sphere $S^{3}$ is said to have Property $\mathbf{P}$ [1] if it is impossible to obtain a simply connected manifold by removing a regular neighborhood of $K$ from $S^{3}$ and sewing it back differently. It has been conjectured that all nontrivial knots have Property $P$, and large classes of knots with this property have been described by Hempel [5], Bing and Martin [1], Noga [10], Connor [2], Gonzales [4], and the author [12]. If $K$ has Property P, then the knot type of $K$ is determined by the topological type of $S^{3}-K$. Furthermore, it would be interesting to know that no fake 3 -sphere could be constructed by surgery along a knot, since [7] any closed, orientable 3-manifold can be realized by surgery along some finite link in $S^{3}$.

In [12], a Property $\mathrm{Q}$ is defined for knots and it is shown there (Theorem 5) that Property $\mathbf{Q}$, along with an additional technical requirement, implies Property $\mathbf{P}$. Property $\mathrm{Q}$ and Neuwirth's notion of an interpolating manifold [9] for a knot are similar in that both require the knot to be contained in a closed 2-manifold in a "sufficiently complicated" manner. It is conjectured in [12] that a knot $K$ has Property $\mathrm{Q}$ iff $K$ has a nontrivial interpolating manifold. This conjecture is established by the following theorem, which is the main result of this paper:

THEOREM. If $S$ is a polyhedral, closed 2-manifold in $S^{3}, K$ a nonseparating simple closed curve in $S$, and $A$ is either closed complementary domain of $S$, then $K$ generates a free factor of $H_{1}(A)$ iff $H_{1}(A, S-K)=0$. It follows that most knots with nontrivial interpolating manifolds have Property $\mathbf{P}$.

Received by the editors May 8, 1970 and, in revised form, February 8, 1971.

AMS 1969 subject classifications. Primary 5520, 5701; Secondary 2022, 5475, 5478, 5560, 5705.

Key words and phrases. Knot, 2-manifold, 3-manifold, interpolating manifold, Property $\mathrm{P}$, homotopy 3-sphere, cube-with-holes, cube-with-handles, Poincaré conjecture.

(1) Research partially supported by NSF Grant GP 19295.

Copyright (C) 1971, American Mathematical Society 
$\$ 1$ contains the proof of the above theorem and its application to Property $\mathbf{P}$. $\S 2$ considers generalizations of the main result: Theorem 2 extends Theorem 1 to knots in manifolds other than $S^{3}$; in Lemma 3.1 necessary criteria are found for an endomorphism of the first homology group of a closed, orientable 2-manifold to be induced by a map. These are used in Theorem 3 to obtain results analogous to Theorem 1 for loops, rather than simple closed curves.

Conventions. All topological spaces, subspaces, and maps considered here are polyhedral, and all manifolds are orientable. A knot is a simple closed curve in $S^{3}$ that does not bound a disk. A manifold is closed if it is compact, connected, and has no boundary. Homology groups are taken with integer coefficients unless otherwise specified. An interpolating manifold for a knot $K$ is a closed 2-manifold $S \subset S^{3}$ such that $K \subset S$ and $K$ does not generate a free factor of the first homology group of either closed complementary domain of $S$; since, as noted in [9], every knot $K$ has an interpolating manifold $S$ such that $K$ separates $S$, we call $S$ nontrivial if $S-K$ is connected. If $K$ is contained in a closed 2-manifold $S$ such that $S-K$ is connected and $S-K$ does not carry the first homology of either closed complementary domain of $S$, then $K$ is said to have Property Q. If $x, y$ are elements of a group $G$, the commutator of $x$ and $y$, denoted $[x, y]$, is $x^{-1} y^{-1} x y$; the commutator subgroup, denoted $G^{\prime}$, of $G$ is the subgroup generated by $\{[x, y]\}_{x, y \in G}$. If $R_{1}, R_{2}, \ldots$ are elements of the free group $F$ generated by $a_{1}, a_{2}, \ldots$, the symbol $P=\left(a_{1}, a_{2}, \ldots\right)$ $R_{1}, R_{2}, \ldots$ ) will denote the quotient group $G$ of $F$ by its smallest normal subgroup containing $R_{1}, R_{2}, \ldots$; if $H$ is a group isomorphic to $G$, then $P$ is called a presentation of $H$ with generators $\left\{a_{i}\right\}$ and (defining) relators $\left\{R_{i}\right\}$.

It will also be useful to define a "standard basis" for a closed 2-manifold $S$ of genus $n \geqq 1$. Let $a_{1}, \ldots, a_{2 n}$ be a system of simple closed curves in $S$ such that (1) if $|i-j|=n$ then $a_{i}$ and $a_{j}$ are transverse, and (2) $a_{i} \cap a_{j}=\varnothing$ otherwise. Choose a base point $s \in S$, and, for $i=1, \ldots, n$, let $t_{i}$ be an arc in $S$ from $s$ to $a_{i} \cap a_{n+i}$ such that for $i \neq j, t_{i} \cap t_{j}=\{s\}$. For $i=n+1, \ldots, 2 n$, let $t_{i}=t_{i-n}$. Orient the curves $a_{i}$, $i=1, \ldots, 2 n$, and let $\alpha_{i}$ be the loop obtained from $a_{i}$ by tracing out $t_{i}, a_{i}$, and then $t_{i}^{-1}$. Then $\left\{\alpha_{i}\right\}_{i=1, \ldots, 2 n}$ generates $\Pi_{1}(S, s)$, and, with possible changes of orientations and renumbering of curves within the pairs $a_{i}, a_{n+i}$, the function $\lambda: \alpha_{i} \rightarrow x_{i}$, $i=1, \ldots, 2 n$, defines an isomorphism of $\Pi_{1}(S, s)$ onto

$$
\left(x_{1}, \ldots, x_{2 n} \mid \prod_{i=1}^{n}\left[x_{i}, x_{n+i}\right]\right) \text {. }
$$

With such orientations and numbering, the curves $\left\{a_{i}\right\}_{i=1, \ldots, 2 n}$ will be called a standard basis for $S$. It may be the case, however, as in $\$ 2$, that necessary properties of $\left\{a_{i}\right\}$ would be lost by renumbering. It is still possible to orient the curves so that $\left[\lambda\left(a_{i}\right), \lambda\left(a_{n+i}\right)\right]$ is conjugate to $\left[x_{i}, x_{n+i}\right]$. With such orientations, $\left\{a_{i}\right\}$ will be called a prestandard basis for $S$. This choice of orientations is independent of the base point $s$ and the arcs $t_{i}$. With any orientations, $\left\{a_{i}\right\}_{i=1, \ldots, 2 n}$ is a basis for $H_{1}(S)$. 
1. The main result. Let $S$ be a closed 2-manifold of genus $n \geqq 1$ in $S^{3}$ containing a nonseparating simple closed curve $K$, and let $A$ be the closure of a complementary domain of $S$.

THEOREM 1. $K \in p H_{1}(A)$ for some prime $p \in Z$ iff there exists a homomorphism of $H_{1}(A, S-K)$ onto $Z_{p}$. In particular, $K$ generates a free factor of $H_{1}(A)$ iff $H_{1}(A, S-K)=0$.

COROllary 1. A knot $K$ has a nontrivial interpolating manifold iff $K$ has Property Q.

COROLlaRY 2. If a knot $K$ has a nontrivial interpolating manifold $S$ such that a boundary component $J$ of a regular neighborhood of $K$ in $S$ is not 0 , a generator, or twice a generator in $H_{1}\left(S^{3}-K\right)$, then $K$ has Property $\mathbf{P}$.

Proof of Corollary 2. By Theorem $1, S$ and $J$ satisfy the requirements of Theorem 5 of [12], and so $K$ has Property $P$.

Proof of Theorem 1. If $n=1$, the result is obvious, so it will be assumed throughout that $n \geqq 2$. Since $A \subset S^{3}$ and bdy $(A)$ is connected, by a theorem of Fox [3], $A$ can be re-embedded in $S^{3}$ so that $\left(S^{3}-A\right)^{-}$is a regular neighborhood of a finite graph. From a theorem of Papakyriakopoulos (Theorem (4.1) of [11]), it then follows that there is a prestandard basis $\left\{a_{i}\right\}$ for $S$ such that

(i) $a_{1}, \ldots, a_{n}$ is a basis for $H_{1}(A)$, a free abelian group of rank $n$,

(ii) $a_{i} \sim 0$ in $A$ for $n+1 \leqq i \leqq 2 n$.

For notational convenience, it will be assumed that no renumbering is necessary to change $\left\{a_{i}\right\}$ to a standard basis for $S$; the arguments below will accommodate any such complication by appropriate alteration of subscripts.

Since $K$ is a nonseparating simple closed curve in $S$, there exists a homeomorphism $f: S \rightarrow S$ taking $a_{1}$ to $K$. Since $f$ is a homeomorphism, $f$ induces a conjugacy class of automorphisms of $\Pi_{1}(S)$, which in turn induces an automorphism $f_{*}$ of $H_{1}(S)$. Let $E=\left(e_{i j}\right)$ be the $2 n \times 2 n$ integer matrix of $f_{*}$, where $f_{*}\left(a_{i}\right)=\sum_{j=1}^{2 n} e_{i j} a_{j}$. By a theorem of Magnus (Corollary 5.15 of [8]), since $f_{*}$ is induced by a homeomorphism, and $\left\{a_{i}\right\}$ is a standard basis, the matrix $E$ must be symplectic. That is, if $I$ is the $n \times n$ identity matrix, $J$ is the $2 n \times 2 n$ matrix with block diagram $\left(\begin{array}{cc}0 & 1 \\ -1 & 0\end{array}\right)$, and $E^{\prime}$ is the transpose of $E$, then $E J E^{\prime}= \pm J$ (although $E J E^{\prime} \neq E^{\prime} J E$ in general, $E$ is symplectic iff $E^{\prime}$ is symplectic). Thus, in particular, for all $s, t$ such that $1 \leqq s<t \leqq 2 n$ and $|s-t| \neq n$, it must be the case that

$$
\sum_{i=1}^{n}\left|\begin{array}{ll}
e_{i, s} & e_{i, t} \\
e_{i+n, s} & e_{i+n, t}
\end{array}\right|=0 .
$$

Since it has been assumed that no renumbering among $a_{i}, a_{i+n}$ was necessary, the remainder of the proof will only make use of equations $(*)$ for $1 \leqq s<t \leqq n$. 
Let $f_{A}: H_{1}(S) \rightarrow H_{1}(A)$ be the homomorphism induced by $f$ followed by inclusion of $S$ into $A$. Then in $H_{1}(A), K=f_{A}\left(a_{1}\right)$ and

$$
\begin{aligned}
H_{1}(A, S-K) & =H_{1}(A) / f_{A} H_{1}\left(S-a_{1}\right) \\
& =H_{1}(A) /\left\{f_{A}\left(a_{i}\right): i=1, \ldots, 2 n, i \neq n+1\right\} .
\end{aligned}
$$

Case 1. Assume that for some prime $p \in Z, K \in p H_{1}(A)$. To map $H_{1}(A, S-K)$ onto $Z_{p}$, it suffices to find a homomorphism of $H_{1}(A)$ onto $Z_{p}$ annihilating $\left\{f_{A}\left(a_{i}\right): i=2, \ldots, 2 n, i \neq n+1\right\}$, since any homomorphism of $H_{1}(A) \rightarrow Z_{p}$ will annihilate $f_{A}\left(a_{1}\right)$.

First define $\sigma: H_{1}(A) \rightarrow H_{1}\left(A ; Z_{p}\right)$ by $\sigma\left(a_{i}\right)=a_{i}$. Then $\sigma f_{A}\left(a_{i}\right)=\sum_{j=1}^{n} e_{i j} a_{j}$, where now $e_{i j}$ is a residue class $\bmod (p)$. The equations $(*)$ remain valid over $Z_{p}$; in particular, since $\sigma f_{A}\left(a_{1}\right)=0$, we have for $1 \leqq s<t \leqq n$,

$$
\sum_{i=2}^{n}\left|\begin{array}{ll}
e_{i, s} & e_{i, t} \\
e_{i+n, s} & e_{i+n, t}
\end{array}\right|=0 \in Z_{p} .
$$

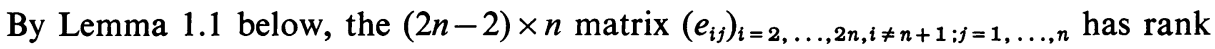
at most $n-1$ over $Z_{p}$. Thus there exists an epimorphism $\theta: H_{1}\left(A ; Z_{p}\right) \rightarrow Z_{p}$ annihilating $\left\{\sigma f_{A}\left(a_{i}\right): i=2, \ldots, 2 n, i \neq n+1\right\}$, and so $\theta \circ \sigma$ induces a map of $H_{1}(A, S-K)$ onto $Z_{p}$.

Case 2. Assume now that for some prime $p \in Z$, there exists an epimorphism $\rho: H_{1}(A) / f_{A} H_{1}\left(S-a_{1}\right) \rightarrow Z_{p}$. We wish to show that $f_{A}\left(a_{1}\right) \in p H_{1}(A)$. Let $\Pi$ be the natural projection of $H_{1}(A)$ onto $H_{1}(A) / f_{A} H_{1}\left(S-a_{1}\right)$, and let $\sigma$ be as in Case 1 . Since $Z_{p}$ has characteristic $p, \theta=\rho \circ \Pi \circ \sigma^{-1}$ is a well-defined homomorphism of $H_{1}\left(A ; Z_{p}\right)$ onto $Z_{p}$. Since $S$ contains generators for $H_{1}(A)$, specifically $a_{1}, \ldots, a_{n}$, and $f$ is a homeomorphism, $f_{A}$ must be surjective, and so $\left\{\sigma f_{A}\left(a_{i}\right): i=1, \ldots, 2 n\right\}$ generates $H_{1}\left(A ; Z_{p}\right)$. Thus it must be the case that $\theta \sigma f_{A}\left(a_{n+1}\right)$ generates $Z_{p}$. On the other hand, if $\sigma f_{A}\left(a_{1}\right) \neq 0$, then by Lemma 1.2 below, equations $(*)$ imply that $\sigma f_{A}\left(a_{n+1}\right)$ is a linear combination of $\left\{\sigma f_{A}\left(a_{i}\right): i=1, \ldots, 2 n, i \neq n+1\right\}$, and so $\theta \sigma f_{A}\left(a_{n+1}\right)=0$. We conclude that $\sigma f_{A}\left(a_{1}\right)=0$, i.e., $K \in p H_{1}(A)$.

LEMMA 1.1. Let

$$
E=\left(e_{i j}\right)_{i=2, \ldots, 2 n, i \neq n+1 ; j=1, \ldots, n}
$$

be a $(2 n-2) \times n$ matrix over a field subject to equations $(* *)$. Then $\operatorname{rank}(E) \leqq n-1$.

Proof. If $n=2$, the result is obvious. We proceed by induction on $n$. The rank of $E$ and equations (**) are preserved under the following transformations: (i) permute columns, (ii) divide a column by a nonzero scalar, (iii) add to one column a multiple of another, and (iv) permute rows in pairs: row $(i) \rightleftarrows$ row $\left(i^{\prime}\right)$ and row $(i+n) \rightleftarrows$ row $\left(i^{\prime}+n\right)$. If rank $(E)=n$, then with finitely many transformations of types (i)-(iv), we can obtain a new matrix $E$, satisfying equations (**), such that $e_{2,1}=e_{2+n, 2}=1$, all the other terms in rows $(i=2)$ and $(i=2+n)$ are 0 , and the submatrix

$$
\bar{E}=\left(e_{i j}\right)_{i=3, \ldots, 2 n: i \neq 1+n, 2+n: j=2, \ldots, n}
$$


has $(n-2)$ linearly independent rows, the first component of each being 0 . But if rows $(i=2)$ and $(i=2+n)$ are as specified, then we have, for $2 \leqq s<t<n$,

$$
\sum_{i=3}^{n}\left|\begin{array}{ll}
e_{i, s} & e_{i, t} \\
e_{i+n, s} & e_{i+n, t}
\end{array}\right|=0,
$$

so inductively, $\operatorname{rank}(\bar{E}) \leqq n-2$. Thus the first component of each row of $\bar{E}$ must be 0 , and so $e_{2+n, 2}=1$ and all the other terms in column $(j=2)$ of $E$ are 0 . Since $e_{21}=1$, this contradicts the equation

$$
0=\sum_{i=2}^{n}\left|\begin{array}{ll}
e_{i, 1} & e_{i, 2} \\
e_{i+n, 1} & e_{i+n, 2}
\end{array}\right| .
$$

LEMMA 1.2. Let $E=\left(e_{i j}\right)_{i=1, \ldots, 2 n ; j=1, \ldots, n}$ be a $2 n \times n$ matrix over a field subject to equations $(*)($ for $1 \leqq s<t \leqq n)$. If row (1) is not identically 0 , then row $(n+1)$ is a linear combination of the other rows of $E$.

Proof. In addition to preserving equations (*), the transformations (i)-(iii) described in the preceding proof do not alter the fact of whether or not row $(n+1)$ of $E$ is a linear combination of the other rows. Since row (1) has some nonzero component, we can therefore assume that row $(1)=(1,0, \ldots, 0)$. Equations $(*)$, for $s=1$, then become

$$
\left\{e_{1+n, t}=\sum_{i=2}^{n}\left|\begin{array}{ll}
e_{i+n, 1} & e_{i+n, t} \\
e_{i, 1} & e_{i, t}
\end{array}\right|\right\}_{t=2, \ldots, n} .
$$

It follows easily that if $\alpha_{j}=e_{j+n, 1},-e_{j-n, 1}$ according as $1 \leqq j \leqq n$ or $n+2 \leqq j \leqq 2 n$, then

$$
\operatorname{row}(n+1)=\sum_{j=1, \ldots, 2 n ; j \neq n+1} \alpha_{j} \cdot \operatorname{row}(j) .
$$

2. Generalizations. Several of the hypotheses of Theorem 1 can be weakened, while maintaining the same or appropriately modified conclusions. First, $p$ need not be prime; Theorem 1 easily extends to the case that $p$ is a product of distinct primes. It is not clear, however, how one might establish a duality theorem of the following sort:

Conjecture. If $K, A, S$ are as in Theorem 1, then the torsion subgroups of $H_{1}(A, K)$ and $H_{1}(A, S-K)$ are isomorphic.

It is also unnecessary to require the ambient space to be $S^{3}$.

Definition. A compact, connected, 3-manifold $A$ with boundary a closed 2-manifold $S$ of genus $n$ is called a homology cube-with-holes (HCWH) if $H_{1}(A)$ is a free abelian group of rank $n$ and $S$ has a prestandard basis $\left\{a_{i}\right\}_{i=1, \ldots, 2 n}$ satisfying conditions (1). From the proof of Theorem 1 we have immediately

THEOREM 2. If $A$ is a HCWH and, otherwise, $K, S, p, A$ are as in Theorem 1 , then $K \in p H_{1}(A)$ iff $\exists \rho: H_{1}(A, S-K) \rightarrow Z_{p}$. 
If $A$ can be embedded in a homology 3-sphere as the closure of the complement of a regular neighborhood of a finite graph, then, from Theorem (4.1) of [11] and the Mayer-Vietoris sequence, it follows that $A$ is a HCWH. If $A$ can be embedded in a homotopy 3-sphere, then, modifying Fox's proof in [3], $A$ can be embedded in a (possibly different) homotopy 3-sphere as the closed complement of a cubewith-handles, so, again, $A$ is a HCWH.

Finally, results analogous to Theorem 1 can be obtained in the case that $K$ is the image of a nonseparating simple closed curve under a map of $S$ to $S$.

THEOREM 3. Let $A$ be a $H C W H$ with boundary $S, K$ a nonseparating simple closed curve in $S, f: S \rightarrow S$ a map, and $f_{A}: H_{1}(S) \rightarrow H_{1}(S) \rightarrow H_{1}(A)$ the induced homomorphism. If, for some prime $p \in Z, f_{A}(K) \in p H_{1}(A)$, then there is a homomorphism of $H_{1}(A) / f_{A} H_{1}(S-K)$ onto $Z_{p}$. The converse holds providing $f_{A}$ is assumed to be surjective.

Proof. Let $E=\left(e_{i j}\right)$ be as in the proof of Theorem 1. The full strength of the fact that $E$ was symplectic was not required for that proof, but only that equations (*) be valid for $1 \leqq s<t \leqq 2 n,|s-t| \neq n$. It thus suffices to verify these equations in the case that $f$ is a map.

Let $\left\{a_{i}\right\}_{i=1, \ldots, 2 n}$ be a prestandard basis for $S, f_{*}\left(a_{i}\right)=\sum_{j=1}^{2 n} e_{i j} a_{j}$ the endomorphism of $H_{1}(S)$ induced by $f$,

$$
x_{s, t}=\sum_{i=1}^{n}\left|\begin{array}{ll}
e_{i, s} & e_{i, t} \\
e_{i+n, s} & e_{i+n, t}
\end{array}\right| \text {, }
$$

and

$$
G_{a, n}=\left(a_{1}, \ldots, a_{2 n} \mid \prod_{i=1}^{n}\left[a_{i}, a_{n+1}\right],\{[u,[v, w]]\}_{u, v, w \in\left\{a_{i}\right\}}\right)
$$

(isomorphic to the quotient group of $\Pi_{1}(S)$ by the third term in its lower central series). Since $f$ is a map and $\left\{a_{i}\right\}$ is a prestandard basis, $f$ induces an endomorphism \# of $G_{a, n}$ which induces $f_{*}$. Thus $\prod_{i=1}^{n}\left[f_{\#}\left(a_{i}\right), f_{\#}\left(a_{n+i}\right)\right]=1 \in G_{a, n}$. But, using the identity $u^{p} v^{q}=v^{q} u^{p}[u, v]^{p q}$ in $G_{a, n}$, it is easy to show that

$$
\begin{aligned}
& \prod_{i=1}^{n}\left[f_{\sharp}\left(a_{i}\right), f_{\#}\left(a_{n+i}\right)\right] \\
& \quad=\left(\prod_{1 \leqq s<t \leqq 2 n:|s-t| \neq n}\left[a_{t}, a_{s}\right]^{x_{s, t}}\right) \cdot\left(\prod_{s=1, \ldots, n-1 ; t=s+n}\left[a_{t}, a_{s}\right]^{x_{s, t}-x_{n, 2 n}}\right) .
\end{aligned}
$$

Since $G_{a, n}^{\prime}$ is a free abelian group, generated by $\left\{\left[a_{t}, a_{s}\right]\right\}_{1 \leqq s<t \leqq 2 n}$ and freely generated by

$$
\left\{\left[a_{t}, a_{s}\right]\right\}_{1 \leqq s<t \leqq 2 n ;(s, t) \neq(n, 2 n)},
$$

it follows that each exponent in the right side of equation (\#) must be 0 .

Question. Which endomorphisms of $H_{1}(S)$ are induced by maps? According to the above calculations, if $E=\left(e_{i j}\right)$ is the matrix of a map-induced endomorphism 
$f_{*}$ of $H_{1}(S)$, given in terms of a prestandard basis for $S$, then $E$ must be "nearly symplectic," in the sense that for some integer $\lambda, E^{\prime} J E=\lambda J$. For genus $(S)=1$, this is no restriction, consonant with the fact that $H_{1}(S)=\Pi_{1}(S)$. But if genus $(S) \geqq 2$, and $f$ is not (homotopic to) a homeomorphism, then, using Euler characteristic arguments, the fact that $\Pi_{1}(S)$ is Hopfian, and Lemma 3.2 of [6], it can be shown that there is a homeomorphism $h: S \rightarrow S$ such that $f \circ h$ annihilates at least one of $a_{i}, a_{n+i}$ for each $i=1, \ldots, n$. It thus follows that $\lambda \neq \pm 1 \Rightarrow \lambda=0$. But is it true that any $2 n \times 2 n$ integer matrix $E$ such that $E^{\prime} J E=0$ is the matrix of a map $f: S \rightarrow S ?$

\section{REFERENCES}

1. R. H. Bing and J. M. Martin, Cubes with knotted holes, Trans. Amer. Math. Soc. 155 (1971), 217-231.

2. A. Connor, Splittable knots, Ph.D. Thesis, University of Georgia, Athens, Ga., 1969.

3. R. H. Fox, On the imbedding of polyhedra in 3-space, Ann. of Math. (2) 49 (1948), 462-470. MR 10, 138.

4. F. Gonzales, On homology spheres, Ph.D. Thesis, Princeton University, Princeton, N. J., 1970.

5. J. Hempel, A simply connected 3-manifold is $S^{3}$ if it is the sum of a solid torus and the complement of a torus knot, Proc. Amer. Math. Soc. 15 (1964), 154-158. MR 28 \#599.

6. W. Jaco, Heegaard splittings and splitting homomorphisms, Trans. Amer. Math. Soc. 144 (1969), 365-379. MR 40 \#6555.

7. W. B. R. Lickorish, $A$ representation of orientable combinatorial 3-manifolds, Ann. of Math. (2) 76 (1962), 531-540. MR 27 \#1929.

8. W. Magnus et al., Combinatorial group theory: Presentations of groups in terms of generators and relations, Pure and Appl. Math., vol. 13, Interscience, New York, 1966. MR 34 \#7617.

9. L. Neuwirth, Interpolating manifolds for knots in $S^{3}$, Topology 2 (1963), 359-365. MR 27 \#6259.

10. D. Noga, Uber den Aussenraum von Produktknoten und die Bedeutung der Fixgruppen, Math. Z. 101 (1967), 131-141. MR 36 \#2137.

11. C. D. Papakyriakopoulos, A reduction of the Poincaré conjecture to other conjectures, Bull. Amer. Math. Soc. 68 (1962), 360-366. MR 26 \#5548.

12. J. Simon, Some classes of knots with property P, Topológy of Manifolds, Markham, Chicago, Ill., 1970, pp. 195-199.

UNIVERSITY OF IOWA, Iowa CITY, Iowa 52240 